\title{
The Coupled Ionosphere and Thermosphere at Mid-Latitudes in the Asian Sector and Its Comparison with Other Locations
}

\author{
Shoichiro FUKAO ${ }^{1}$, Tomoyuki TAKAMI ${ }^{1}$, and William L. OLIVER ${ }^{2}$ \\ ${ }^{1}$ Radio Atmospheric Science Center, Kyoto University, Japan \\ ${ }^{2}$ Department of Electrical, Computer and Systems Engineering and Center for Space Physics, \\ Boston University, U.S.A.
}

(Received March 10, 1995; Revised October 21, 1995; Accepted December 13, 1995)

\begin{abstract}
The present paper reviews dynamical features of the mid-latitude ionosphere and thermosphere in the Asian sector as quantified by the MU radar at Shigaraki, Japan $\left(35^{\circ} \mathrm{N}, 136^{\circ} \mathrm{E}\right)$ and compares these with dynamics reported for other locations. The thermospheric wind and temperature in the Asian sector differ from the wind and temperature measured at other mid-latitude locations. Furthermore, the perpendicular plasma drifts have strong resemblances to those predicted by current models, but their seasonal trends suggest a strong conjugate effect that has not been fully incorporated into our expectations of local behavior. These results provide additional evidence that global features cannot be described by a simple zonal mean. Finally, two features related to gravity waves are discussed. Gravity waves seem to be ubiquitous in the thermosphere and play an important role in creating a variety of electron density fluctuations and irregularities in the mid-latitude ionosphere. Tracking the progression of ionospheric gravity-wave effects between MU radar beams has allowed gravity wave packets to be identified and their dispersion relation to be determined. The character of ionospheric irregularities observed are consistent with their seeding by the Perkins mechanism.
\end{abstract}

\section{Introduction}

The Japanese MU (Middle and Upper atmosphere) radar is the newest of the large atmospheric radars capable of detecting the incoherent scatter from free electrons in the ionosphere. It has accumulated data for about eight years, and its data base is now large enough to permit various climatological analyses of the ionosphere and thermosphere. The thermosphere is controlled mainly by solar heating, which depends on geographic latitude, whereas the ionosphere is controlled mainly by the geomagnetic field, which depends on geomagnetic latitude. The concept of a zonal mean model is not applicable: owing to the displaced geographic and geomagnetic poles, the thermosphere-ionosphere coupling is not the same at all longitudes. The present paper reviews dynamical features of the mid-latitude ionosphere and thermosphere in the Asian sector as quantified by the MU radar at Shigaraki, Japan $\left(35^{\circ} \mathrm{N}, 26^{\circ} \mathrm{N}\right.$ geomagnetic, conjugate point $18^{\circ} \mathrm{S}$ ) and compares these with the dynamics reported primarily by other radars, such as the Arecibo radar $\left(18^{\circ} \mathrm{N}, 30^{\circ} \mathrm{N}\right.$ geomagnetic, conjugate point $\left.45^{\circ} \mathrm{S}\right)$.

\section{Thermospheric Meridional Winds and Temperature}

Recently, the MU radar has been used to make the first incoherent scatter radar measurements of neutral meridional winds in the Asian sector (Oliver et al., 1990). These winds are highly diurnal and generally consistent with mid-latitudes winds measured elsewhere concerning the phases and times of wind direction turnings. The amplitudes of the MU radar winds, however, were noted by Oliver et al. (1990) to be generally smaller by a factor of 1.5-2 than wind amplitudes measured elsewhere. More recent wind climatologies (Duboin and Lafeuille, 1992; Hagan, 1993) are showing values and daily trends much closer 
to the MU results, except for some specific time intervals during the day. The MU wind results have also been confirmed recently by winds estimated from ionograms collected in Japan (Igi et al., 1995). At the MU radar location we see no trace of an abatement in the southward wind at midnight, like that seen at lower latitudes (Arecibo), nor of strong winds exceeding $200 \mathrm{~m} / \mathrm{s}$ at night, like those seen near the polar regions (Millstone Hill). The MU radar wind is not consistent with the mean wind or the diurnal variation of the HWM90 model (Hedin et al., 1991).

The seasonal patterns of electron density and electron and ion temperatures have been combined with a theory of ionosphere-thermosphere heat exchange to infer the behavior of the neutral temperature in the $F$ region (Oliver et al., 1991). The neutral temperature over Shigaraki is consistently lower than that predicted by current models by amounts ranging up to $150 \mathrm{~K}$. The size of this difference depends on solar activity and season, being much larger in spring and summer than in fall and winter. But this study did not have an abundance of data: when all data (August 1986 to April 1990) were sorted into 24 one-hour bins, 4 season bins, and 2 solar-activity bins, the bin populations ranged from 0 to 6 measurements.

\section{Perpendicular Plasma Drifts}

During the process of collecting and analyzing several years of MU radar ionospheric $F$ region drift data, we have come to recognize patterns of behavior that we have formed into a set of rules, perhaps yet incomplete and not without exception, to explain these drifts: (1) the daytime electric fields are mapped up from $E$ region dynamo fields, (2) the nighttime fields are $F$ region dynamo fields generated by $F$ region winds, (3) sunrise and sunset drift enhancements are due to relative conductivity changes in the $E$ and $F$ layers, and (4) all of the aforementioned effects are controlled jointly by conditions in the local and magnetic conjugate hemispheres. The following data will illustrate these patterns.

For our discussions in this section of the paper, in which we address only the drifts perpendicular to the magnetic field lines, we will drop the prefix "perpendicular".

\subsection{Solar cycle variations}

Figure 1 shows the average behavior of the eastward velocities, separated into summer, winter, and equinox seasons and into periods of low and high solar activity. These data have been averaged over three hours to eliminate the short-term perturbations which we address later in this paper. A striking aspect of the eastward drifts is that there is little change in the daytime drifts over the solar cycle whereas there is marked change in the nighttime drifts. This simple fact leads us to the basic belief that the daytime drifts, being insensitive to solar activity, are driven by processes originating in lower parts of the atmosphere, where solar-activity variations are weak, whereas nighttime drifts, being sensitive to solar activity, are driven by processes in the upper portions of the atmosphere, where solar-activity variations are strong. This explanation, however, is incomplete if we assign the daytime drifts to the $E$ region dynamo mechanism and the nighttime drifts to the $F$ region dynamo mechanism. MU radar measurements show that $F$ region winds are somewhat weaker at solar maximum while the $F$ region drifts shown in Fig. 1 are much stronger. They also show that there is no late-evening reversal of the summer wind at solar minimum to correspond with the reversal of the drifts shown in Fig. 1. A possible interpretation of these data is that the daytime data have mostly a semi-diurnal character (12-hour period) while the nighttime data undergo transition from a largely diurnal character (24-hour period) at solar maximum to a more semi-diurnal character at solar minimum, this latter pattern being especially evident in summer. If we then associate the semi-diurnal variation with the $E$ region winds (semi-diurnal tidal propagation) and the diurnal variation with the $F$ region winds (in-situ tide, which we noted above to be very diurnal in character), this transition could mean that a more complicated sharing of electric-field control develops between the $E$ and $F$ regions at night as solar activity decreases, because both the neutral and electron densities (and hence the effectiveness of dynamo action) decrease with decreasing solar activity much faster in the $F$ region than in the $E$ region. We comment further on $E$ and $F$ region control of the ionospheric electric field later in this report. 
Eastward Perpendicular Drift
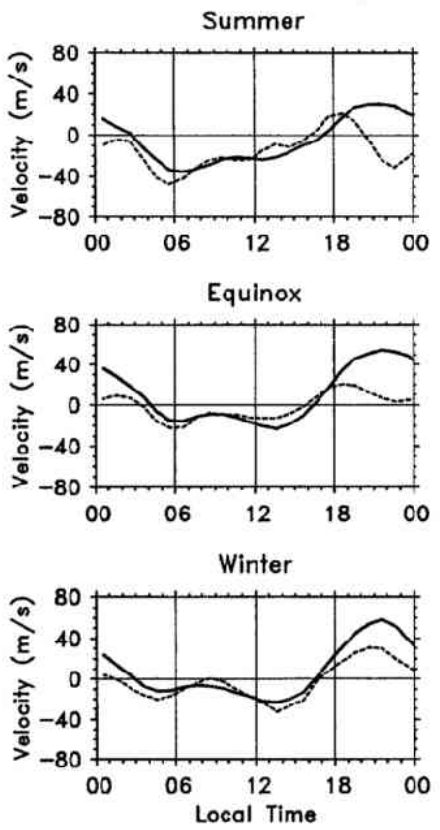

Fig. 1. Average perpendicular-eastward drifts measured by the MU radar during quiet times $(K p<3+)$ at low (dashed line) and high (solid line) solar activity.

\subsection{Conjugate effects}

We have identified strong magnetic-conjugate influences on the drifts measured by the MU radar, not through direct information about conditions at the $\mathrm{MU}$ conjugate point $\left(18^{\circ} \mathrm{S}\right)$ but through comparison with drifts measured at Arecibo $\left(18^{\circ} \mathrm{N}\right)$, which happens to have the same latitude (distance from the equator) as the MU conjugate point, but in the opposite hemisphere. Figures 2 and 3 show the MU and Arecibo radar drifts in summer and winter at solar maximum. It is clear that the MU summer curves are similar to the Arecibo winter curves and that the MU winter curves are similar to the Arecibo summer curves. Since Arecibo and the MU conjugate point have the same latitude, they should have the same average seasonal winds and hence the same average seasonal dynamo generation of electric fields. This would result in the resemblance between the drifts above Arecibo and the MU conjugate point six months apart, in opposite seasons, e.g., Arecibo June summer solstice should resemble MU-conjugate-point December summer solstice. We have found this hypothesis to be valid but to represent only half of the story: conductivity comparison represents a controlling factor in this similarity relationship (Takami et al., 1996b).

A simple electric circuit analogue aids our explanation. Let us restrict ourselves for the moment to the ideal nighttime case in which the $E$ region is absent and the $F$ region dynamo generates the electric fields. Let the dynamo process in one of two conjugate $F$ regions be represented by a voltage source $V_{1}$ and let the resistance of that $F$ region be represented by a resistor $R_{1}$. The conjugate hemisphere will have corresponding elements $V_{2}$ and $R_{2}$. These circuit elements are connected in series by the zero-resistance magnetic field lines. The net voltage $V$ experienced by the charged particles in either $F$ region is the voltage across the sourcc-resistor combination in that region, and, by Kirchhoff's voltage law, the net voltage must be the same for each hemisphere. $V$ will range between its extreme values of $V_{1}$ and $V_{2}$, depending on the relative values of $R_{1}$ and $R_{2}$. If $R_{1}=R_{2}$, half the voltage difference will be dropped across each resistor, 


\section{Eostward Perpendicular Drift}

$\mathrm{F} 10.7>120$
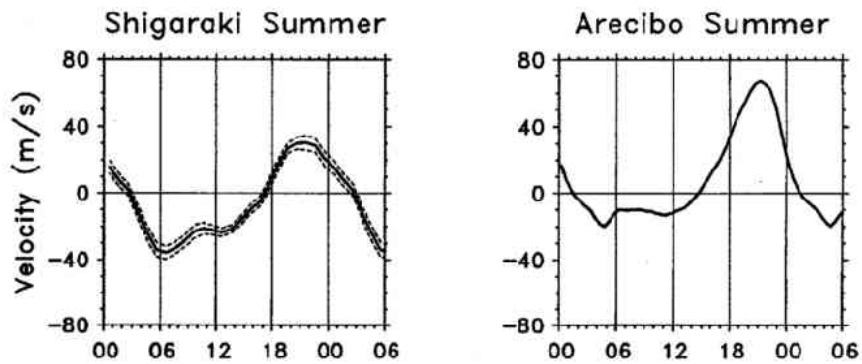

Shigaraki Winter

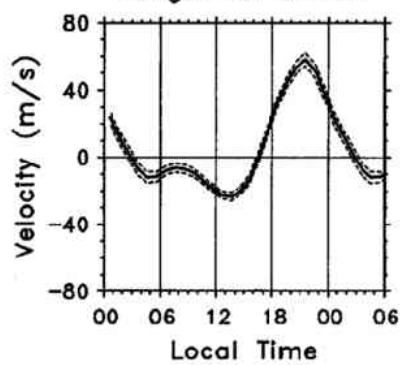

Arecibo Winter

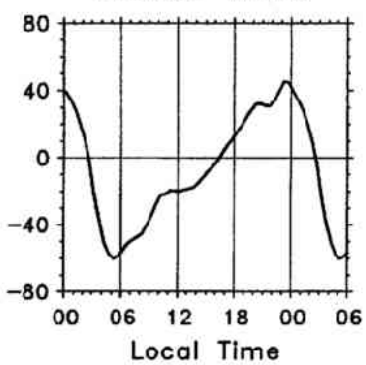

Fig. 2. Average perpendicular-eastward drifts measured with the MU and Arecibo radars during summer and winter at solar maximum. The dashed curves show the range of uncertainty in the MU radar average curve.

\section{Northward Perpendicular Drift}

$\mathrm{F} 10.7>120$

Shigaraki Summer

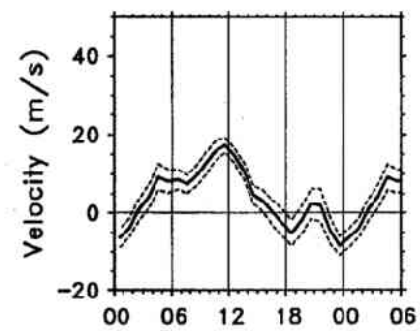

Arecibo Summer

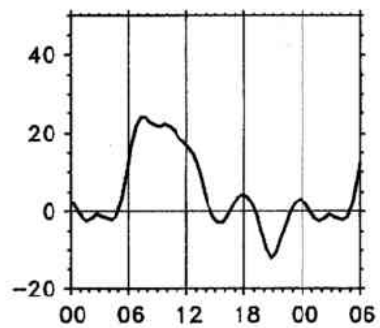

Shigaraki Winter
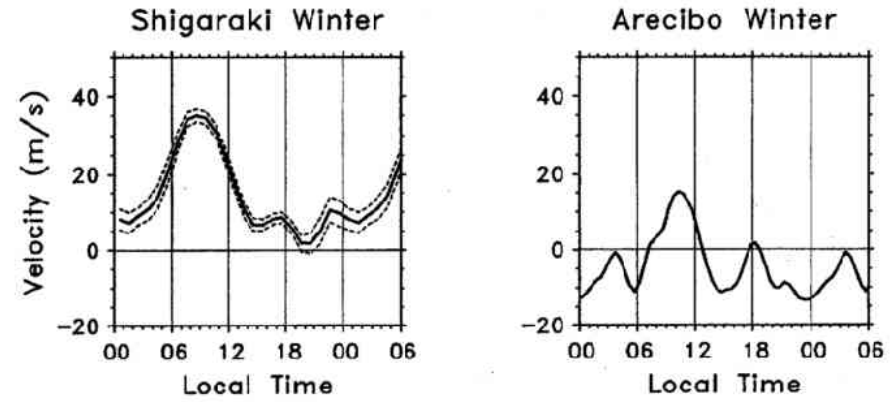

Fig. 3. Average perpendicular-northward drifts measured with the MU and Arecibo radars during summer and winter at solar maximum. The dashed curves show the range of uncertainty in the MU radar average curve. 
and each hemisphere will experience the same voltage $V=\left(V_{1}+V_{2}\right) / 2$; the dynamos effects are averaged. If $R_{1}$ is much smaller than $R_{2}$, however, $R_{1}$ will drop no voltage, $R_{2}$ will drop the entire $V_{2}-V_{1}$ voltage difference, and each hemisphere will experience the voltage $V_{1}$. In this case, hemisphere 2 is absolutely slaved to hemisphere 1: perpendicular drifts in hemisphere 2 are determined solely by the dynamo field in hemisphere 1.

Now let us apply this picture to the Arecibo-MU comparison, looking first at the MU-winter Arecibosummer case. The Arecibo drifts will be controlled by some average of $F$ region dynamo voltages at Arecibo and at its own conjugate point (remember that we are restricting ourselves to the nighttime for the moment). The MU drifts likewise will be controlled by some average of $F$ region dynamo voltages at Shigaraki and at its own conjugate point. Below we show the latitudes and local seasons for these four places.

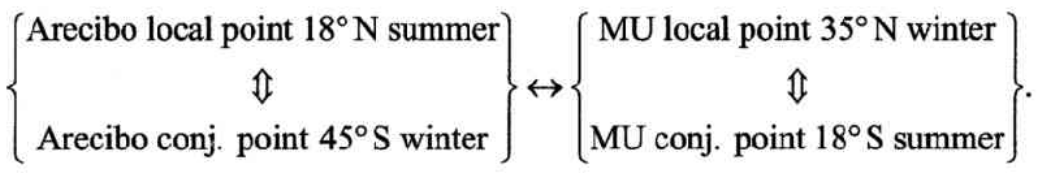

Since the MU winter nighttime drifts are so similar to the Arecibo summer nighttime drifts, it must be that the dynamos and conductivities at Arecibo and its conjugate location combine to give the same net ionospheric voltage $V$ as do the dynamos and conductivities at the MU location and its conjugate point. For this to happen the following three hypotheses must be true:

1. The Arecibo and MU-conjugate locations, having the same latitude, also have the same average wind patterns in their respective summer seasons.

2. The conductivity at Arecibo is much greater than the conductivity at the Arecibo conjugate point.

3. The conductivity at the MU conjugate point is much greater than the conductivity at the MU location.

The first hypothesis allows equal dynamo voltages to be generated at Arecibo and at the MU conjugate point. The second and third hypotheses ensure that these dynamos (at Arecibo and at the MU conjugate point) dominate over the dynamos generated at their conjugate locations (at the Arecibo conjugate point and at the MU location) so that the voltages impressed on the ionospheres at all four locations are the same and equal to the Arecibo dynamo voltage. We have little direct data to check the validity of these hypotheses, but it does seem reasonable that locations at $18^{\circ} \mathrm{N}$ and $18^{\circ} \mathrm{S}$ have similar average summer winds. Hypotheses 2 and 3 require that the low-latitude summer locations have much higher electron densities than the mid-latitude winter conditions at night, and both the latitude and seasonal conditions work in our favor here. The seasonal condition is probably the more important. While the electron density is largest in winter during the daylight hours (because of the higher neutral atomic oxygen density), the opposite is true at night. The reason for this is clear: in summer the meridional winds turn equatorward to push the ionosphere up the field lines to altitudes of higher equilibrium density before sunset occurs, and maintains this lift all night long, keeping this high density in a region where the decay is slow. In winter, on the other hand, the winds are still pushing the ionosphere down the field lines to regions of lower equilibrium density when sunset occurs, and at these low altitudes this winter ionosphere decays rapidly until the winds finally turn to raise what little is left of that once-strong winter density to a higher altitude where the decay is slower. The resulting ratio of summer/winter $F$ region densities is large enough to give the summer hemisphere dynamo a controlling influence over the winter hemisphere dynamo.

We have concentrated our attention so far on the Arecibo-summer MU-winter eastward drifts because their similarity is the most outstanding in our plots. For the Arecibo-winter MU-summer case in Fig. 2 the local seasons at the four locations are switched: 


$$
\left\{\begin{array}{c}
\text { Arecibo local point } 18^{\circ} \mathrm{N} \text { winter } \\
\Uparrow \\
\text { Arecibo conj. point } 45^{\circ} \mathrm{S} \text { summer }
\end{array}\right\} \leftrightarrow\left\{\begin{array}{c}
\text { MU local point } 35^{\circ} \mathrm{N} \text { summer } \\
\Uparrow \\
\text { MU conj. point } 18^{\circ} \mathrm{S} \text { winter }
\end{array}\right\} .
$$

The key to what happens is the conductivities, and if we stick with our reasonable hypothesis that the summer hemisphere will have the larger conductivity, then we find that the dynamo control in this case is exerted by Arecibo's conjugate point and the MU local point, just the opposite from the previous case. But these controlling points are now at different latitudes, $35^{\circ}$ and $45^{\circ}$, so the Arecibo-winter and MUsummer drifts should be similar only to the extent that the winds at $35^{\circ}$ and $45^{\circ}$ are similar. We expect that this is the reason for the obviously poorer similarity in the Arecibo-winter MU-summer case.

Our reasoning should apply as well for the northward drifts, and Fig. 3 shows that the same AreciboMU seasonal matches occur here as they did for the eastward drifts but that the similarity is substantially weaker. The MU northward drifts cannot yet be understood as simply as the eastward drifts can.

During solar minimum (Figs. 4 and 5), we see the same similarities for the eastward drifts as we did for solar maximum, but the correspondence has weakened considerably, while for the northward drift we cannot see any such similarities at all. This deterioration of the similarities could mean that one or more of the three hypotheses that we set forth to explain the solar maximum case has become less valid-the dynamo winds at the two controlling locations may become less similar or dominance in conductivity at one of the two conjugate locations may fail, meaning that there are no controlling locations. It could also mean that our neglect of the $E$ region is no longer appropriate. The electron density does decrease toward solar minimum much more markedly in the $F$ region than in the $E$ region, and the $E$ region may then come to have an important effect on the $F$ region even at night.

\section{Eastward Perpendicular Drift \\ F10.7 $<120$}
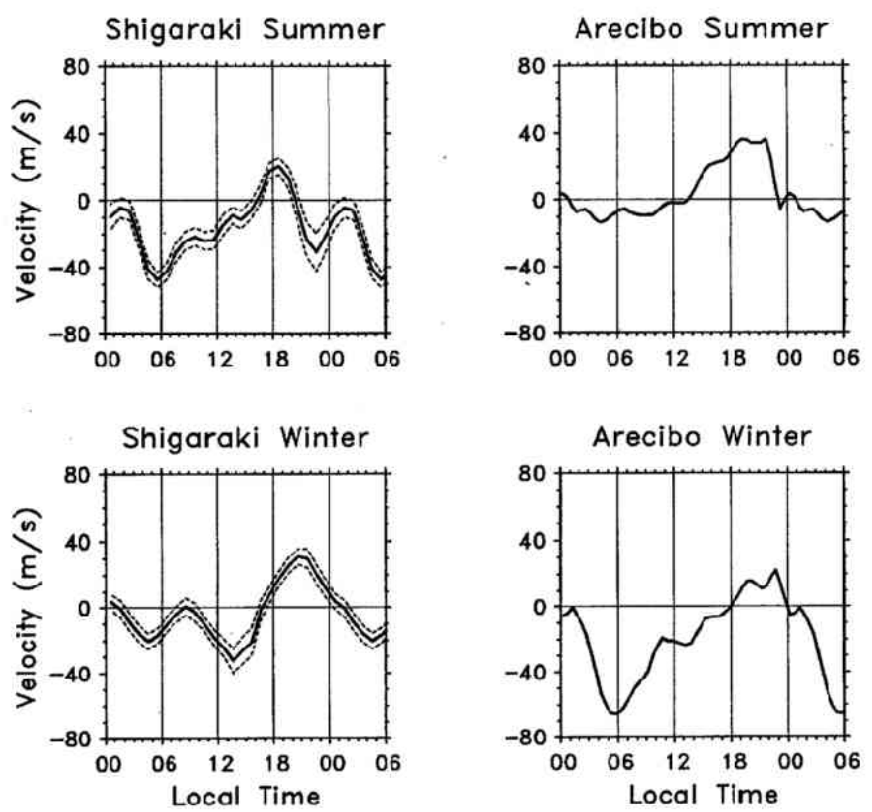

Fig. 4. Average perpendicular-eastward drifts measured with the MU and Arecibo radars during summer and winter at solar minimum. The dashed curves show the range of uncertainty in the MU radar average curve. 
The circuit picture used above to explain the nighttime case can be extended to the daytime case by adding an $E$ region source-resistor combination in parallel with each $F$ region source-resistor combination. Until recently the accepted picture was that during sunlit hours the $E$ region conductance was always much greater than the $F$ region conductance, so the $F$ region was not important in electric-field generation. The competition for control would then be between conjugate $E$ regions, and, for the middle and low latitudes under consideration here, the $E$ region electron density is photochemically controlled and a wellbehaved function of solar-zenith angle. Thus during daytime the summer hemisphere should tend to have larger influence than the winter hemisphere but should not totally dominate. This picture changed recently when Buonsanto (1994) showed that during solar maximum the electron and neutral densities in the $F$ region increase to such an extent that the $F$ region conductance can exceed the $E$ region conductance during daytime in winter, though not by a commanding ratio. This would mean that during daytime at solar maximum the winter hemisphere would have the larger influence, though it would not totally dominate. Thus daytime electric fields should switch from a greater summer hemisphere influence to a greater winter hemisphere influence as solar activity rises, but in general without commanding control by either hemisphere and, at solar maximum, with two $E$ regions and two $F$ regions all contributing in an important way.

These considerations would show up in our drift plots as follows. If the summer hemisphere were dominant, the better similarity would be between Arecibo-summer and Shigaraki-winter. If the winter hemisphere were dominant, the reverse would be true. Looking at Fig. 2, at the daytime hours, we believe that it is true that, for solar maximum daytime, the Arecibo-winter Shigaraki-summer similarity is stronger. This implies a larger winter influence, in line with Buonsanto's finding of greater $F$ region than $E$ region influence. For solar minimum, we would expect to see the greater influence pass to the $E$ region during the daytime, and hence to the summer hemisphere, resulting in a stronger Arecibo-summer

\section{Northward Perpendicular Drift \\ $\mathrm{F} 10.7<120$}
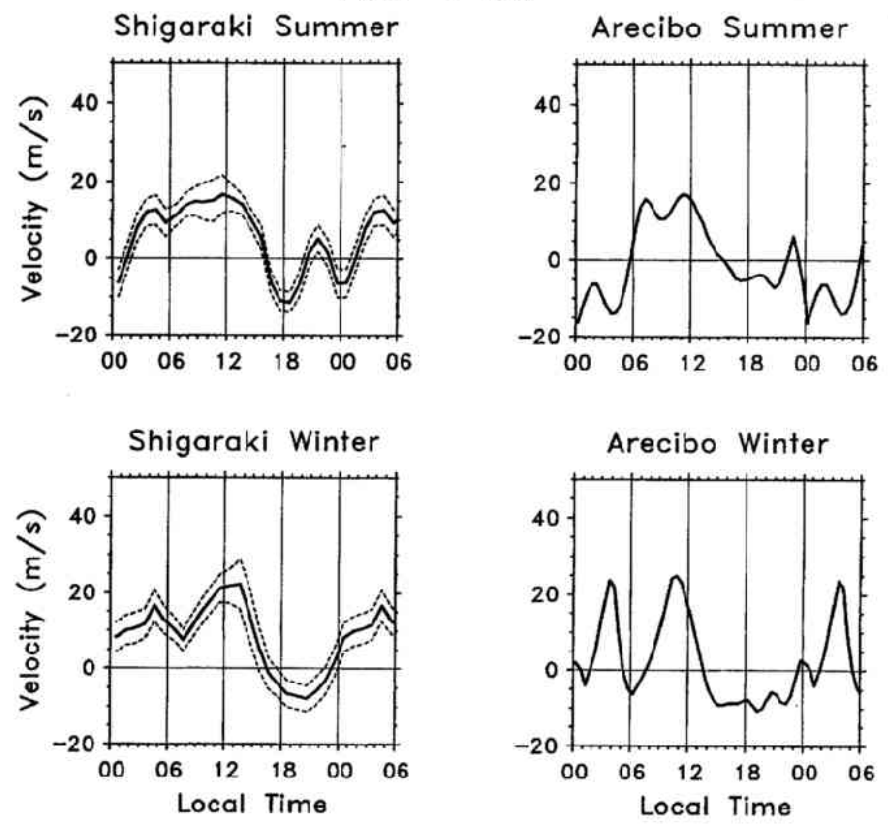

Fig. 5. Average perpendicular-northward drifts measured with the MU and Arecibo radars during summer and winter at solar minimum. The dashed curves show the range of uncertainty in the MU radar average curve. 
Shigaraki-winter similarity. Our judgement from Fig. 4, however, is that the better similarity is once again between Arecibo-winter and Shigaraki-summer, or a retained winter hemisphere primary influence. We do not think that the winter $E$ region would be stronger than the summer $E$ region, but we wonder if the "Ganguly effect" might possibly come into play here. Ganguly et al. (1987) made the important point, with computational confirmation, that even if the conductances should be the same in the $E$ and $F$ regions, the $F$ region may remain the dominant generator of the dynamo voltage. This is because the $F$ region winds are stronger, for one thing, and, more importantly, because the $F$ region can make a greater contribution to the voltage generation function, which is an integral of the product of wind and conductivity over altitude, since the height-wise oscillatory character of the $E$ region winds diminishes the potential $E$ region contribution whereas the height-independent character of the $F$ region winds makes fully additive contributions. So it may still be that the winter $F$ region would retain the stronger influence during the daytime at solar maximum.

While some of the points made above are speculative, they do serve to point out the variety of considerations necessary to gain an understanding of these complicated dynamics and also to point out the futility of approaching this topic from the viewpoint of local processes alone.

\section{Evening Enhancement of the Zonal Electric Field}

The evening enhancement of the upward plasma drift, often called the postsunset or prereversal enhancement, is a well known phenomenon in the equatorial $F$ region (e.g., Fejer et al., 1989). Having reviewed previous works, Takami et al. (1996a) have noticed that the evening upward enhancement is followed immediately by a downward enhancement, whereas in the morning sector a downward enhancement is followed by an upward enhancement. The enhancements are observed in all seasons during high and middle solar activity but during low solar activity are perceptible only in the evening sector at equinox. The occurrence times and amplitudes of the evening enhancements have a clear seasonal variation. According to Takami et al. (1996a) the basic features of the enhancements can be explained on the basis of the model of Farley et al. (1986). It seems that $F$ region eastward winds build up, through dynamo action, unipolar charge accumulations in the dusk and dawn sectors, and thesecharge accumulations create the electric fields that cause the enhancements. These $F$ region charges flow freely through the $E$ region during the daylight hours of high $E$ region conductivity and hence cannot accumulate to form strong electric fields. But as the $E$ region conductivity falls rapidly at sunset the $F$ region charges accumulate rapidly, and the dynamo control of the ionospheric electric field is transferred from the $E$ region to $F$ region.

The same model may be applied to temperate latitudes, since it incorporates no specific parameter related to the equatorial ionosphere. The MU radar data show that such enhancements indeed occur at mid latitudes in the Asian sector, although their magnitudes are not so large as those in the equatorial regions. This may indicate that the enhancements are a universal phenomenon occurring all over the globe. A number of factors lead us to believe that these enhancements are caused by conductivity changes rather than rapid wind turnings. The first is that the winds driving these drifts are known to be, on average, highly diurnal whereas the excursions develop over a much shorter period. The second is that both the Shigaraki and the Arecibo data show that the enhancements occur simultaneously in the eastward and northward drifts and that Arecibo and Shigaraki experience enhancements of the same sense and at the same time but in opposite seasons (indicating conjugate electric-field effects, as discussed earlier). And the third feature is that, although these enhancements are rather broad, covering several hours, they are unlikely to be produced by background winds both because the winds have predominantly 24 - and 12 -hour periods and because wind models show that the north-south and east-west wind components have different phases (inconsistent with the observation of simultaneous enhancements in the northward and eastward ionospheric drifts). 


\section{Gravity Waves}

By observing the ionospheric $F$ region simultaneously in multiple beams with the MU radar, it is possible to track the passage of gravity waves and measure the horizontal electron density gradients that they produce (Fukao et al., 1993). We have verified through simulation that the gradient structure and fluctuations are consistent with a gravity wave source which shows wave amplitude decay in the direction perpendicular to the direction of propagation. It appears that the ionosphere is essentially always perturbed by gravity waves to a degree detectable with an incoherent scatter radar. These waves appear to exist over a broad range of periods and frequencies. The period-frequency relation is, on average, consistent with the linear dispersion relation for gravity waves but from day to day can vary greatly, even on two successive days that are magnetically extremely quiet. Figure 6 shows all of the daily-average results so far available from the MU radar ionospheric gravity-wave experiment, together with a straight line fitted to each day's data. The data between 200 and $300 \mathrm{~km}$ altitude have been averaged for these displays. Figure 7 shows these data averaged to display full-year and seasonal trends. We see that on average the wave speed is essentially constant at $200 \mathrm{~m} / \mathrm{s}$ for periods from 60 to 130 minutes. This speed is close to the value expected from linear asymtotic wave theory for a lossless medium. Only mild seasonal effects in the

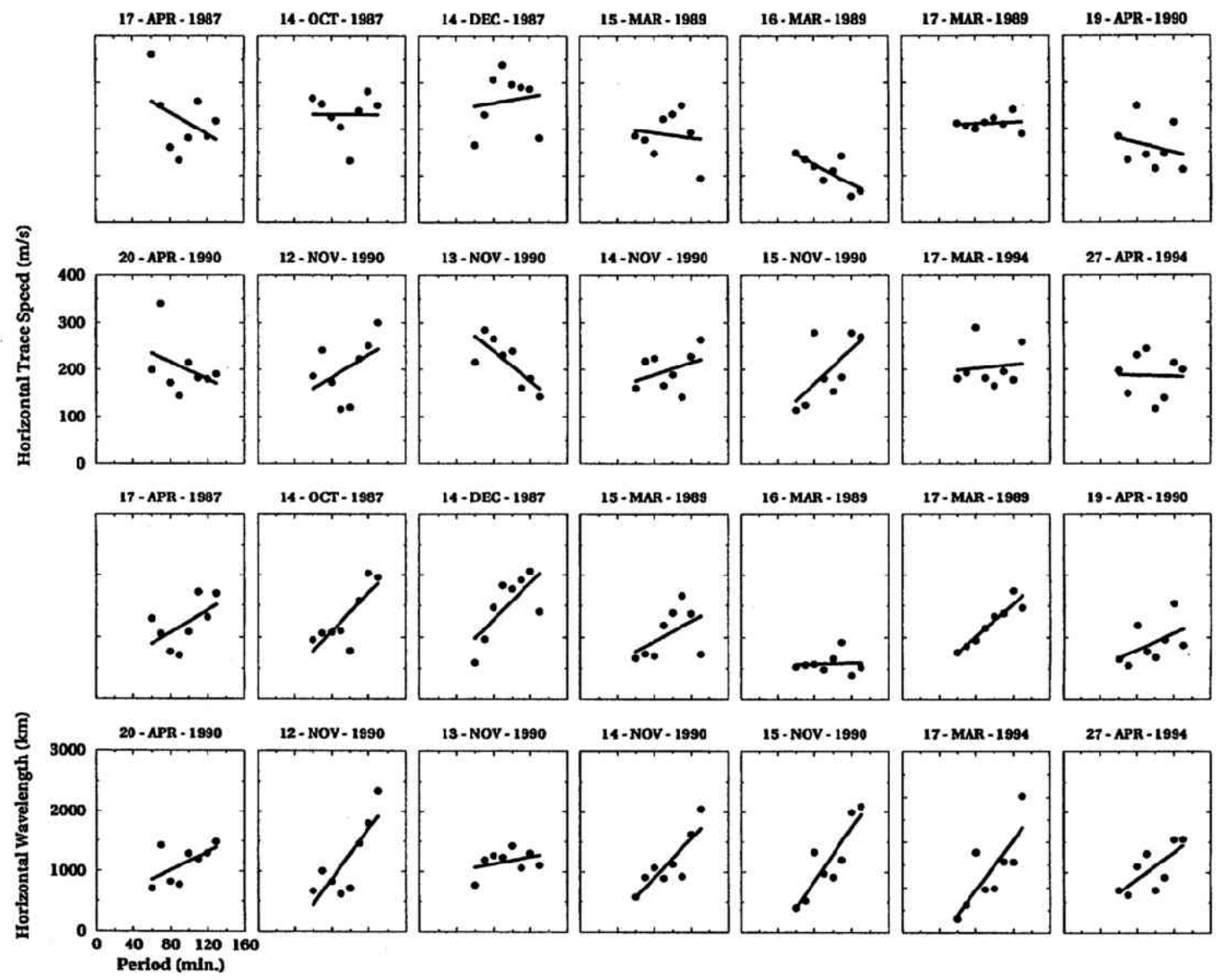

Fig. 6. Gravity wave horizontal speed and wavelength versus period for 14 days of observation. The data were collected during the daytime hours $08-16 \mathrm{LT}$ and were averaged over 200-300 km altitude. 

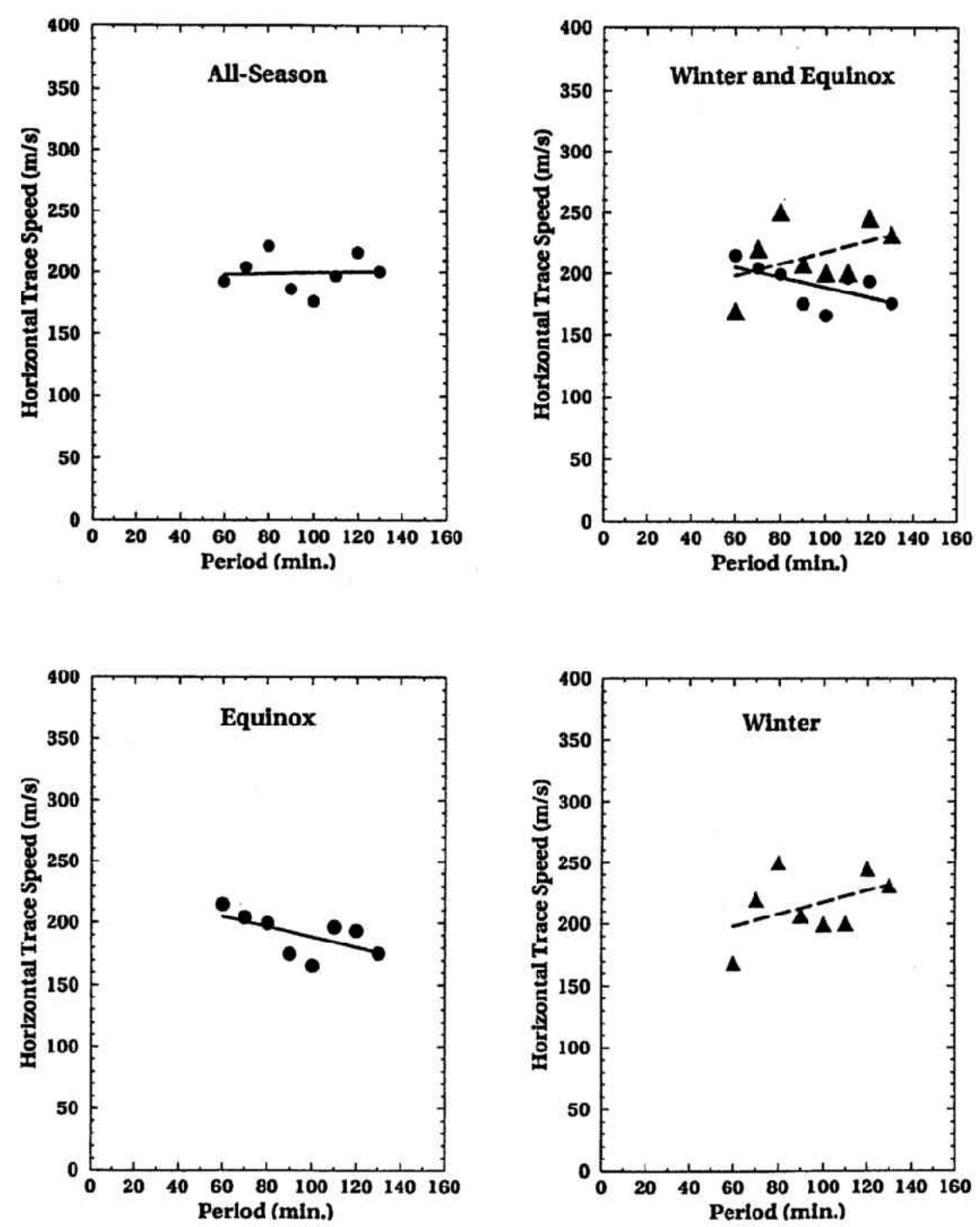

Fig. 7. The data of Fig. 6 averaged in different ways: all-season, equinox, winter, and equinox and winter superposed.

dispersion relation are seen. These data (plus data from work in progress) have failed to identify any seasonal variation or magnetic-activity variation in gravity-wave propagation except for an indication that the wave speed is reduced by $50 \mathrm{~m} / \mathrm{s}$ for wave periods between 60 and 100 minutes during extremely quiet times and that the propagation direction is significantly more southward on quiet days. The latter characteristic may be evidence for a filtering out of waves travelling in the same direction as the background wind. The waves persist for only one or two cycles, and apparently longer trains seem to result from fortuitous temporal juxtaposition of independent waves. Although the propagation direction and speed of most wave packets are constant throughout their transit over the radar, there are cases in which the speed or the direction or both change, or else another wave (at the same frequency) transits at the same time, yielding complicated density signatures that are difficult to interpret. The great day-to-day variability in the wave characteristics suggests that the wave propagation is very sensitive to conditions, unknown to us, in the lower parts of the atmosphere. 


\section{Ionospheric Irregularities}

The MU radar has found that during sunspot minimum the mid-latitude ionosphere over Japan is sometimes characterized by regions of rapid and turbulent upwelling (Fukao et al., 1991). The most likely cause of this upwelling seems to be, as was proposed by Perkins (1973), the instability of the equilibrium which occurs when the mid-latitude plasma is supported against gravity either by an eastward electric field or by a southward neutral wind. The growth rate determined by Perkins is considerably higher at sunspot minimum than at sunspot maximum for comparable altitudes of the ionospheric $F$ layer. The growth rate is not very large, however, and the observed structures must evolve from preexisting undulations on the bottomside of the $F$ region, undulations that are generated by gravity waves. That is, the gravity waves create finite amplitude structures which are amplified by the plasma instability (Kelley and Fukao, 1991).

Yamamoto et al. (1991) observed intense echoes generated by ionospheric irregularities in the $E$ region. They found a distinctive feature in the echoes during the post-sunset period in summer: these echoes were classified as "quasi-periodic" because their appearance was intermittent with a period of 510 minutes. The quasi-periodic echoes show a fairly constant phase propagation toward the radar while the average background Doppler velocity is away from the radar. Using multibeam observations with the MU radar, Yamamoto et al. (1994) have showed an apparent westward motion of the echoes. In the postsunrise period, on the other hand, the echoes have appeared continuously at altitudes of $90-100 \mathrm{~km}$ altitude and have been labeled as the "continuous" type (Ogawa et al., 1995). Yamamoto et al. (1992) also reported that both types of echoes occur more often in summer than in winter.

There are now two models proposed for the generation of quasi-periodic echoes in the $E$ region. Woodman et al. (1991) proposed that they can be generated by the gradient-drift instability when the altitude of sporadic- $E$ layers are modulated (as a function of horizontal distance) by short-period gravity waves. They suggested that gravity waves with phase fronts parallel to the magnetic field are capable of producing such distortion, imposing their own temporal and spatial periodicity on the echoes. In this case the altitude extent of the modulation was expected to be only a few kilometers for each sporadic $E$ layer. Tsunoda et al. (1994) re-examined the model and proposed a much larger modulation, up to $\pm 15 \mathrm{~km}$, and showed that such modulation would lead to the development of polarization electric fields. Both of these models invoke specific geometries for quasi-periodic echo layers as a favorable condition for interaction with propagating gravity waves. Knowledge of the actual spatial distribution of the irregularities and their transport characteristics is important for model validation. In May-July 1993, Yamamoto et al. (1996) conducted simultaneous observations with the MU radar and the Frequency Agile Radar, installed about $40 \mathrm{~km}$ north of Shigaraki, and found that the quasi-periodic echo regions propagated southwestward.

\section{Conclusion}

We have reviewed several dynamical features of the mid-latitude ionosphere and thermosphere in the Asian sector as observed by the MU radar. Some features related to global structure, such as neutral temperature, meridional neutral wind, and perpendicular plasma drifts, show appreciable differences from existing model values. The enhancements super-imposed on the smooth diurnal variation of the drifts seem to occur on a global scale, to be caused by transitions between $E$-region and $F$-region dynamo control associated with conductivity changes, and to evidence strong conjugate effects. The MU radar measurements indicate that internal gravity waves are ubiquitous in the thermosphere and that they cause electron density fluctuations and remarkably intense irregularities in both the $E$ and $F$ regions. These features suggest that the structure and dynamics of the ionosphere and thermosphere over Japan are significantly different from those observed elsewhere.

The MU radar is owned and operated by the Radio Atmospheric Science Center of Kyoto University. WLO was partially supported in this work by grant ATM-9321491 from the National Science Foundation of the U.S.A. 


\section{REFERENCES}

Buonsanto, M. J., Evidence for polarization electric fields in the daytime $F$ region above Millstone Hill, J. Geophys. Res., 98, 6437-6446, 1994.

Duboin, M.-L. and M.Lafeuille, Thermospheric dynamics above Saint-Santin: Statistical study, J. Geophys. Res.,97, 8661-8671, 1992.

Farley, D. T., E. Bonelli, B. G. Fejer, and M. F. Larsen, The prereversal enhancement of the zonal electric field in the equatorial ionosphere, J. Geophys. Res., 91, 13723-13728, 1986.

Fejer, B. G., F region plasma drifts over Arecibo: Solar cycle, seasonal, and magnetic activity effects, J. Geophys. Res., 98, 13645$13652,1993$.

Fejer, B. G., E. R. de Paula, I. S. Batista, E. Bonclli, and R. F. Woodman, Equatorial $F$ region vertical plasma drifts during solar maxima, J. Geophys. Res., 94, 12049-12054, 1989.

Fukao, S., M.C. Kelley, T. Shirakawa, T. Takami, M. Yamamoto, T. Tsuda, and S. Kato. Turbulent upwelling of the mid-latitude ionosphere: 1. Observational results by the MU Radar, J. Geophys. Res., 96, 3725-3746, 1991.

Fukao, S., Y. Yamamoto, W. L. Oliver, T. Takami, M. D. Yamanaka, M. Yamamoto, T. Nakamura, and T. Tsuda, Middle and upper atmosphere radar observations of ionospheric horizontal gradients produced by gravity waves, J. Geophys. Res., 98, 9443-9451, 1993.

Ganguly, S., R. A. Behnke, and B. A. Emery, Average electric field behavior in the ionosphere above Arecibo, J. Geophys. Res., 92, 1199-1210, 1987.

Hagan, M. E., Quiet time upper thermospheric winds over Millstone Hill between 1984 and 1990, J. Geophys. Res., 98, 3731$3739,1993$.

Hedin, A. E., M. A. Biondi, R. G. Burnside, G. Hernandez, R. M. Johnson, T. L. Killeen, C. Mazaudier, J. W. Meriwether, J. E. Salah, R. J. Sica, R. W. Smith, N. W. Spencer, V. B. Wickwar, and T. S. Virdi, Revised global model of thermosphere winds using satellite and ground-based observations, J. Geophys. Res., 96, 7657-7688, 1991.

Igi, S., W. L. Oliver, T. Ogawa, and S. Fukao, Comparisons of thermospheric meridional winds derived from $F_{2}$ region peak heights with the winds derived by the MU radar, J. Geophys. Res., 100, 21323-21326, 1995.

Kelley, M. C. and S. Fukao, Turbulent upwelling of the mid-latitude ionosphere: 2. Theoretical framework, J. Geophys. Res., 96, 3747-3753, 1991.

Ogawa, T., M. Yamamoto, and S. Fukao, MU radar observations of turbulence and movement of mid-latitude sporadic $E$ irregularities, J. Geophys. Res., 100, 12173-12188, 1995.

Oliver, W. L., S. Fukao, T. Takami, M. Yamamoto, T. Tsuda, T. Nakamura, and S. Kato, Thermospheric meridional winds measured by the middle and upper atmosphere radar, J. Geophys. Res., 95, 7683-7692, 1990.

Oliver, W. L., T. Takami, S. Fukao, T. Sato, M. Yamamoto, T. Tsuda, T. Nakamura, and S. Kato, Measurements of ionospheric and thermospheric temperatures and densities with the MU radar, J. Geophys. Res., 96, 17827-17838, 1991.

Oliver, W.L., Y. Yamamoto, T. Takami, S. Fukao, M. Yamamoto, and T. Tsuda, Middle and upper atmosphere radar observations of ionospheric electric fields, J. Geophys. Res., 98, 11615-11627, 1993.

Perkins, F. W., Spread $F$ and ionospheric currents, J. Geophys. Res., 78, 218-226, 1973.

Takami, T., W. L. Oliver, and S. Fukao, Interpretations of the equatorial evening/morning enhancements of the $F$ region zonal electric field, J. Atmos. Terr. Phys., 1996a (in revision).

Takami, T., W. L. Oliver, and S. Fukao, Ionospheric drift similarities atmagnetic conjugate and non-conjugate locations, J. Geophys. Res., 1996b (in revision).

Tsunoda, R. T., S. Fukao, and M. Yamamoto, On the origin of quasi-periodic radar backscatter from mid-latitude sporadic $E$, Radio Sci., 29, 349-365, 1994.

Woodman, R. F., M. Yamamoto, and S. Fukao, Gravity wave modulation of gradient drift instabilities in mid-latitude sporadic E irregularities, Geophys. Res. Lett., 18, 1197-1200, 1991.

Yamamoto, M., S. Fukao, T. Tsuda, S. Kato, and R. F. Woodman, Mid-latitude $E$-region field-aligned irregularities observed with the MU Radar, J. Geophys. Res., 96, 15943-15949, 1991.

Yamamoto, M., S. Fukao, T. Ogawa, T. Tsuda, and S. Kato, A morphological study on mid-latitude $E$-region field-aligned irregularities observed with the MU radar, J. Atmos. Terr. Phys., 54, 769-777, 1992.

Yamamoto, M., N. Komoda, S. Fukao, R. T. Tsunoda, T. Ogawa, and T. Tsuda, Spatial structure of the E-region field-aligned irregularities revealed by the MU Radar, Radio Sci., 29, 337-347, 1994.

Yamamoto, M., S. Fukao, R. T. Tsunoda, K. Igarashi, and T. Ogawa, Preliminary results from joint measurements of $E$-region field-aligned irregularities using the MU Radar (MUR) and the Frequency-Agile Radar (FAR), J. Atmos. Terr. Phys., 1996 (in revision). 